\title{
A potentially crucial role of the PKD1 C-terminal tail in renal prognosis
}

\author{
Eiji Higashihara $^{1}$ - Shigeo Horie ${ }^{5}$ Moritoshi Kinoshita $^{6}$ Peter C. Harris ${ }^{7}$. \\ Takatsugu Okegawa ${ }^{2}$ Mitsuhiro Tanbo $^{2} \cdot$ Hidehiko Hara $^{2}$ ' Tsuyoshi Yamaguchi ${ }^{2}$. \\ Kaori Shigemori $^{2} \cdot$ Haruna Kawano $^{5}$ Isao Miyazaki $^{3} \cdot$ Shinya Kaname $^{4}$. \\ Kikuo Nutahara ${ }^{2}$
}

Received: 12 June 2017/ Accepted: 25 August 2017/Published online: 5 October 2017

(c) The Author(s) 2017. This article is an open access publication

\begin{abstract}
Background Autosomal dominant polycystic disease (ADPKD) often results in renal failure. Recently, allelic influences of $P K D 1$ mutation types on renal survival were extensively investigated. Here, we analyzed integrated influences of $P K D 1$ mutation types and positions on renal survival.

Methods We included 338 (82 pedigrees) and 72 (12 pedigrees) patients with $P K D 1$ and $P K D 2$ mutations,
\end{abstract}

Electronic supplementary material The online version of this article (doi:10.1007/s10157-017-1477-7) contains supplementary material, which is available to authorized users.

Eiji Higashihara

ehigashi@ks.kyorin-u.ac.jp

1 Department of ADPKD Research, Kyorin University School of Medicine, 6-20-2 Shinkawa, Mitaka, Tokyo 181-8611, Japan

2 Department of Urology, Kyorin University School of Medicine, Tokyo, Japan

3 Department of Radiology, Kyorin University School of Medicine, Tokyo, Japan

4 Department of Nephrology, Kyorin University School of Medicine, Tokyo, Japan

5 Department of Urology, Juntendo University School of Medicine, Tokyo, Japan

6 Diagnostic Division, Development Department, Otsuka Pharmaceutical Co., Ltd, Tokushima, Japan

7 Division of Nephrology and Hypertension, Mayo Clinic, Rochester, MN, USA respectively, identified through comprehensive gene analysis of 101 probands with ADPKD. Genetic testing was performed using next-generation sequencing, long-range PCR, and multiplex ligation-dependent probe amplification. Pathogenic mutations were identified by a software package-integrated seven databases and provided access to five cloud-based computing systems.

Results Mean renal survivals of carriers with PKD1 nontruncating-type mutations at positions upstream of G-protein-coupled receptor proteolytic site (GPS-upstream domain), transmembrane domain, or cytoplasmic C-terminal tail (CTT) domain were 70.2, 67.0, and 50.1 years, respectively $(P<0.0001)$; renal survival was shorter for mutation positions closer to CTT domain, suggesting its crucial role in renal prognosis. Furthermore, in truncatingtype mutations, strong inactivation is anticipated on nucleotides downstream from the mutation site, implying CTT domain inactivation irrespective of mutation site. Shorter mean renal survival was found for $P K D 1$ truncating-type than non-truncating-type mutation carriers $(P=0.0348)$; mean renal survival was not different between $P K D 13^{\prime}$ - and $5^{\prime}$-region truncating-type mutation carriers $(P=0.4375)$, but was shorter in $P K D 13^{\prime}$-region than in $5^{\prime}$-region non-truncating-type mutation carriers $(P=0.0014)$. Variable strength of CTT domain inactivation might account for these results.

Conclusions Aforementioned findings indicate that CTT domain's crucial role in renal prognosis needs further investigation by larger studies (ClinicalTrials.gov; NCT02322385).

Keywords Autosomal dominant polycystic kidney disease (ADPKD) - Genotype/phenotype correlation · PKD1

mutation $\cdot$ Renal survival 


\section{Introduction}

Autosomal dominant polycystic kidney disease (ADPKD) is the most common genetic kidney disease that leads to end-stage renal disease (ESRD) by the age of 60-70 years in approximately $50 \%$ of patients [1-4]. Approximately 85 and $15 \%$ of patients develop ADPKD due to PKDl and $P K D 2$ mutations, respectively [5-10]. Accumulating evidence indicates that these genes represent the most powerful determinant of disease severity in patients with ADPKD; survival to ESRD is 15-20 years less in patients with $P K D 1$, than in those with $P K D 2$ mutations $[8,11]$.

$P K D 1$ mutations have significant allelic influences on renal phenotypic expression. The influence of truncating-type $P K D 1$ mutations was reported to be more severe than that of nontruncating-type $P K D l$ mutations in renal survival studies $[8,12]$, and in an estimated glomerular filtration rate (eGFR) study [10]. Allelic influences of PKD1 mutations on heightadjusted total kidney volume (htTKV) were observed only after the in silico division of non-truncating-type mutations [10]. The position of $P K D 1$ mutations (in the $5^{\prime}$ - versus $3^{\prime}$-region) has been suggested to correlate with a renal phenotype including earlier mutations, resulting in an increased severity of the disease [13]. However, the effect of the position of PKDI mutations has not been reported in other large studies $[8,10]$.

PKDl encodes polycystin-1 (PC1), a 460-kDa protein with a large extracellular $\mathrm{N}$-terminal region, a transmembrane region, and a cytoplasmic C-terminal tail (CTT) [14, 15]. PKD2 encodes polycystin-2 (PC2), a member of the transient receptor potential family of non-selective cation channels. PC1 and PC2 interact through their CTT domains, and colocalize to the primary cilium, where they may perform mechanosensory functions [16]. The ciliary trafficking of $\mathrm{PC} 1$, and the formation of the PC1-PC2 complex, are regulated by the PC1-PC2 interaction $[17,18]$. PC1 undergoes cleavage at its G-protein-coupled receptor proteolytic site (GPS), that is probably essential for its full function [19]. The CTT region of PC1 may also undergo cleavage, with the resulting CTT being translocated to the nucleus, where it initiates signaling [20,21].

Few genotype/phenotype correlation studies have focused on the structure of PC1. Therefore, the results of the present study might contribute to our understanding of domain influences on $P K D l$ mutations.

\section{Materials and methods}

\section{Study design}

Integrated allelic influences of the position and type of $P K D 1$ mutations on renal survival were examined in 410 patients with documented $P K D 1$ or PKD2 mutations.

\section{Participants}

The study recruited 101 probands with ADPKD (age $>20$ years) who visited the Kyorin University Hospital (KUH; $N=82$ ) and the Juntendo University Hospital (JUH; $N=19$ ) in Tokyo, between January 2014 and October 2015. ADPKD was diagnosed using previously described criteria [22]. Basic data for survival analysis were collected from pedigree members. The onset of ESRD was defined as the initiation of renal replacement therapy (RRT). The non-ADPKD subjects (control) included in this study were second-degree relatives of patients with ADPKD.

Informed consent was obtained from each participant. The study protocol adhered to the Declaration of Helsinki, and was approved by the institutional review boards of KUH and JUN (nos. 579 and 14-043, respectively). This study is registered in ClinicalTrials.gov, under accession no. NCT02322385.

\section{Mutation analysis}

The detection and pathogenicity prediction of missense mutations were performed using next-generation sequencing (NGS), and long-range polymerase chain reaction (LRPCR); data analysis was performed using software packages, as previously described [23]. Multiplex ligation-dependent probe amplification (MLPA) was used to analyze large genomic rearrangements, if pathogenic mutations were not detected by NGS.

PC1 and PC2 structural domains were classified using the Universal Protein Resource (UniProt) annotation [24] of UCSC, and a published study [25].

\section{Classification of PKD1 mutation types and mutation strength groups}

PKD mutations were classified into truncating- and nontruncating-type mutations. Truncating-type mutations included frameshift mutations, nonsense mutations, canonical splicing mutations, in-frame indels of $\geq 5$ amino acids, and large rearrangements; non-truncating-type mutations included missense mutations, in-frame indels of $\leq 4$ amino acids, and non-canonical splicing mutations [10].

PKD1 mutations were classified into mutation strength groups (MSGs), according to previously reported methods [10]. Truncating-type PKD1 mutations were defined as MGS1. Non-truncating-type $P K D 1$ mutations were further divided into strongly predicted mutations (MGS2), and less strongly predicted mutations (MSG3), by using similar criteria, such as substitution scores [5]. 


\section{Classification of PKD1 mutation bisection positions and domain positions}

Two bisection positions were selected at nucleotide \#7978, as an equal group of patients in the present study $\left(5^{\prime}\right.$-segment: \#1-\#7978; 3'-segment: \#7979-\#12,912), and at nucleotide \#6456 as a cDNA midpoint (5'-segment: nucleotide positions \#1-\#6456; 3'-segment: nucleotide positions \#6457-\#12,912).

PKD1 mutation positions were classified into three domains, using nucleotide positions: the GPS-upstream domain, \#1-\#9183; transmembrane domain, \#9223\#12,318 and the CTT domain, \#12,319-\#12,909.

\section{Statistical analyses}

Parametric variables are expressed as mean \pm standard deviation or standard error. Effects of covariates (genotypes, mutation types, and mutation positions) on the cumulative probability of renal and general survival were analyzed using the Kaplan-Meier method, and the univariate Cox's proportional hazards model. For renal survival analysis, living subjects not undergoing RRT at the time of the study and dead subjects without RRT were considered as censored subjects, while those undergoing RRT were considered as non-censored subjects. In noncensored subjects, the survival year indicated the age at which the subject had started RRT. For general survival analysis, the survival year indicated the age at the inclusion in the study for living subjects (censored subjects), or the age of death for dead subjects (non-censored subjects).

The effects of covariates on continuous and categorical variables were examined using an analysis of variance, and Pearson's Chi-squared test, respectively. The hazard ratio (HR) is shown, with a $95 \%$ confidence interval (CI).

All statistical analyses were performed using JMP ${ }^{\circledR}$ ver. 10.0.0 Basic Analysis and Graphing (SAS Institute Inc., Cary, NC). All the tests were two-sided, and $P<0.05$ was considered statistically significant.

\section{Results}

\section{Distribution of pathogenic PKD1 and PKD2 mutations}

Likely pathogenic ADPKD mutations were identified in 94 of 101 families (mutation detection rate, 93.1\%), of which 82 families (81.2\%) had $P K D 1$, and 12 families (11.9\%) had PKD2 mutations. Truncating-type mutations were detected in $59.8 \%$ families with $P K D 1$, and in $75.0 \%$ of families with $P K D 2$ mutations. Using MLPA, we detected large rearrangements in four patients, in whom NGS did not detect pathogenic mutations (Table 1). Sixty pathogenic mutations were newly described in 65 pedigrees, of which 57 were $P K D 1$, and $8 P K D 2$ mutations (Supplemental Table 1). These novel mutations have been described elsewhere [23].
Table 1 Distribution of pathogenic $P K D 1$ and $P K D 2$ mutations

\begin{tabular}{lll}
\hline Gene/mutation type & Pedigrees, $n(\%)$ & \\
\hline PKD1 & $82(81.2 \%)$ & \\
Truncating mutation & $49(59.8 \%)$ & 29 \\
Frameshift & & 10 \\
Nonsense & & 6 \\
Splice (canonical) & $33(40.2 \%)$ & 4 \\
Large rearrangements & & 26 \\
Non-truncating mutation & & 4 \\
Missense & & 3 \\
Splice (non-canonical) & & \\
Inframe change $(<4$ amino acids) & $9(75.0 \%)$ & 4 \\
PKD2 & & 3 \\
Truncating mutation & & 2 \\
Frameshift & & 3 \\
Nonsense & & \\
Splice & & $3(25.0 \%)$ \\
Non-truncating mutation & & \\
Missense & & \\
Mutation negative pedigrees & & \\
\hline
\end{tabular}


Table 2 Demographic characteristics of subjects used for survival analyses

\begin{tabular}{|c|c|c|c|c|c|}
\hline & \multirow{2}{*}{$\begin{array}{l}\text { Non-ADPKD family } \\
\text { member }\end{array}$} & \multirow{2}{*}{$\begin{array}{l}\text { Patients with } P K D 1 \\
\text { mutation }\end{array}$} & \multirow{2}{*}{$\begin{array}{l}\text { Patients with } P K D 2 \\
\text { mutation }\end{array}$} & \multicolumn{2}{|c|}{ Pearson's Chi-square test } \\
\hline & & & & $\begin{array}{l}\text { Three } \\
\text { groups }\end{array}$ & $\begin{array}{l}\text { PKD1 vs } \\
\text { PKD2 }\end{array}$ \\
\hline Number of pedigrees & 71 & 82 & 12 & - & - \\
\hline $\begin{array}{l}\text { Subjects used for survival } \\
\text { analyses }\end{array}$ & 301 & 338 & 72 & - & - \\
\hline Men/women & $151 / 150$ & $159 / 179$ & $35 / 37$ & 0.7325 & 0.8086 \\
\hline Alive/dead & $238 / 63$ & $196 / 142$ & $40 / 32$ & $P<0.0001$ & 0.7045 \\
\hline $\mathrm{RRT}(-) /(+)$ & $300 / 1$ & $214 / 124$ & $64 / 8$ & $P<0.0001$ & $P<0.0001$ \\
\hline Paternal/maternal/unknown & - & $99 / 149 / 90$ & $25 / 31 / 16$ & - & 0.5948 \\
\hline
\end{tabular}

$R R T$ renal replacement therapy

\section{Demographic characteristics of subjects included in survival analyses}

Demographic data for 301 non-ADPKD family members, 338 patients with $P K D 1$ mutations, and 72 patients with $P K D 2$ mutations are presented in Table 2.

Affected and unaffected members of families with ADPKD showed non-significant differences with respect to gender distribution $(P=0.7325)$. However, survival and renal survival differed significantly between these groups $(P<0.0001$ for both; Table 2$)$. Renal survival also differed between patients with $P K D 1$ and $P K D 2$ mutations $(P<0.0001$, Table 2).

\section{Genic effect}

Mean renal survival was shorter by 8.4 years in patients with $P K D 1$ mutations, than in those with PKD2 mutations $(P<0.0001$; Table 3; Supplemental Fig. 1). Life survival was also significantly different between non-PKD family members, and patients with PKD1 and PKD2 mutations $(P<0.0001$; Supplemental Table 2; Fig. 1).

\section{Allelic influences of PKD1 mutations on renal survival}

\section{Influences of PKD1 mutation types and MSGs}

Carriers of truncating-type $P K D I$ mutations displayed shorter renal survival than carriers of non-truncating-type PKD1 mutations, according to the Kaplan-Meier analysis (log rank test, $P=0.0348$; Table 3; Fig. 2), and Cox's proportional hazard analysis $(P=0.0365$; Table 3$)$.

The influence of PKDI MSGs on renal survival was not significant (Table 3; Supplemental Fig. 2). However, renal survival times tended to be longer in patients in the MSG3 group than in those in the MSG1 group (log rank test, $P=0.0605$; Wilcoxon test, $P=0.0437$, according to the
Kaplan-Meier analysis, and $P=0.0573$, according to Cox's proportional hazard analysis).

Influences of the positions of PKD1 mutations, according to mutation types

The influences of the positions of PKD1 mutations were analyzed separately for truncating- and non-truncating-type mutations, because their effects on the structure of $\mathrm{PC} 1$ were assumed to differ, according to mutation types. Truncatingtype mutations affect all downstream amino acid sequences from the mutated position, whereas non-truncating-type mutations are expected to only weakly or locally affect downstream amino acid sequences, although they may alter the folding and localization of the protein [17].

Bisection positions were selected at nucleotide \#7978 (Table 3) and \#6456 (Supplemental Table 3), as explained in the "Materials and methods". The effects of the bisection positions on renal survival were examined in three groups, namely, in the truncating-plus non-truncating-type (all), truncating-type, and non-truncating-type mutation groups.

In the truncating-type and truncating- plus non-truncating-type mutation groups, renal survival did not differ between patients with mutations in the $5^{\prime}$ - and $3^{\prime}$-regions, divided at nucleotide \#7978. In contrast, in the non-truncating-type mutation group, renal survival was shorter in carriers of mutations in the $3^{\prime}$-region than in carriers of mutations in the $5^{\prime}$-region (log rank test, $P=0.0014$; Table 3; Fig. 3). Qualitatively similar differences were also confirmed between the $3^{\prime}$ - and $5^{\prime}$-positions which were divided at nucleotide \#6456 (Supplemental Table 3).

The influence of non-truncating-type mutation positions on renal survival was further examined by dividing the position of the mutations into the GPS-upstream domain, the transmembrane domain, and the CTT domain. Renal survival was longer in the above order (log rank test, $P=0.0001$; Table 3; Fig. 4), and Cox's hazard analysis 
Table $3 P K D$ genic and $P K D 1$ allelic influences on renal survival

\begin{tabular}{|c|c|c|c|c|c|c|c|}
\hline \multirow{2}{*}{$\begin{array}{l}\text { Genic and allelic } \\
\text { variables }\end{array}$} & \multirow{2}{*}{$\begin{array}{l}\text { Subjects } \\
(n)\end{array}$} & \multicolumn{3}{|c|}{ Renal survival (years) by Kaplan-Meier analysis } & \multicolumn{3}{|c|}{ Cox's proportional hazards analysis } \\
\hline & & Mean & SE & $P$ value & $\begin{array}{l}\text { Univariate } \\
\text { HR }\end{array}$ & $95 \% \mathrm{CI}$ & $P$ value \\
\hline \multicolumn{8}{|l|}{$P K D$ genic influence } \\
\hline$P K D 1$ & 338 & 66.87 & 0.98 & Log rank test, $P<0.0001$ & 6.8 & $3.54-15.18$ & $<0.0001$ \\
\hline$P K D 2$ & 72 & 75.22 & 0.72 & Wilcoxon test, $P<0.0001$ & 1 (referent) & & \\
\hline \multicolumn{8}{|c|}{$P K D 1$ allelic influence } \\
\hline \multicolumn{8}{|l|}{ PKD1 mutation type } \\
\hline Non-truncating & 134 & 69.14 & 1.50 & Log rank test, $P=0.0348$ & 1 (referent) & & \\
\hline Truncating & 204 & 64.81 & 1.22 & Wilcoxon test, $P=0.0595$ & 1.47 & $1.02-2.14$ & 0.0365 \\
\hline \multicolumn{8}{|c|}{ PKD1 mutation strength group (MSG) } \\
\hline MSG 1 & 204 & 64.81 & 1.22 & Log rank test, $P=0.1072$ & 1.50 & $0.95-2.47$ & 0.0864 \\
\hline MSG 2 & 75 & 65.33 & 1.55 & Wilcoxon test, $P=0.1174$ & 1.03 & $0.58-1.85$ & 0.9131 \\
\hline MSG 3 & 59 & 69.82 & 2.06 & & 1 (referent) & & \\
\hline \multicolumn{8}{|c|}{ PKD1 mutation type and position } \\
\hline \multicolumn{8}{|l|}{ All $P K D 1$ mutations } \\
\hline $5^{\prime}$-end position & 174 & 67.49 & 1.28 & Log rank test, $P=0.2263$ & 1 (referent) & & \\
\hline $3^{\prime}$-end position & 164 & 65.69 & 1.42 & Wilcoxon test, $P=0.2027$ & 1.24 & $0.87-1.77$ & 0.2346 \\
\hline \multicolumn{8}{|c|}{ Truncating-type $P K D 1$ mutations } \\
\hline $5^{\prime}$-end position & 125 & 64.01 & 1.53 & Log rank test, $P=0.4375$ & 1 (referent) & & \\
\hline $3^{\prime}$-end position & 79 & 65.30 & 1.94 & Wilcoxon test, $P=0.8920$ & 0.84 & $0.52-1.32$ & 0.4452 \\
\hline \multicolumn{8}{|c|}{ Non-truncating-type $P K D 1$ mutations } \\
\hline $5^{\prime}$-end position & 49 & 71.29 & 1.54 & Log rank test, $P=0.0014$ & 1 (referent) & & \\
\hline $3^{\prime}$-end position & 85 & 65.11 & 1.91 & Wilcoxon test, $P=0.0041$ & 2.72 & $1.46-5.38$ & 0.0013 \\
\hline \multicolumn{8}{|c|}{ Non-truncating-type $P K D 1$ mutations } \\
\hline $\begin{array}{l}\text { GPS-upstream } \\
\text { domain }\end{array}$ & 56 & 70.17 & 1.49 & $\begin{array}{l}\text { Log rank test, } P=0.0001 \\
\text { Wilcoxon test, } P<0.0001\end{array}$ & 1 (referent) & & \\
\hline $\begin{array}{l}\text { Transmembrane } \\
\text { domain }\end{array}$ & 66 & 67.01 & 2.19 & & 1.94 & $1.05-3.68$ & 0.0354 \\
\hline CCT domain & 12 & 50.07 & 1.67 & & 6.61 & $2.32-16.53$ & 0.0010 \\
\hline
\end{tabular}

5'-region position: nucleotide position \#1-\#7978; 3'-region position: nucleotide position \#7979-\#12,912. GPS-upstream domain: nucleotide position \#1-\#9183; Transmembrane domain: nucleotide position \#9223-\#12,318; CCT domain: nucleotide position \#12,319-\#12,909

GPS G-protein-coupled receptor proteolytic site, $C C T$ cytoplasmic C-terminal tail

confirmed a significant difference between each of the domains (Table 3). In truncating-type mutations, however, no significant difference was observed on renal survival among three domains (log rank test, $P=0.6551$ according to the Kaplan-Meier analysis, and $P=0.6447$ according to Cox's proportional hazards analysis).

\section{Allelic influences of PKD1 mutations on survival}

PKD1 mutation types did not have a significant influence on survival (Supplemental Table 2). Carriers of $5^{\prime}$-end mutations showed longer survival times than those of $3^{\prime}$ end mutations, when the groups were divided at nucleotide \#6456, but the difference was not significant when divided at nucleotide \#7978. In non-truncating-type mutation carriers, survival was shorter in $3^{\prime}$-end mutation carriers than in $5^{\prime}$-end mutation carriers (log rank test, $P=0.0053$; Supplemental Table 2). Survival was also significantly different among carriers of PKDl non-truncating-type mutations in the GPS-upstream domain, the transmembrane domain, and the CTT domain, and it became shorter in this order (log rank test, $P=0.0036$; Supplemental Table 2).

\section{Effects of sex and parental origin}

Women showed a trend of longer renal survival than men; however, the difference was not statistically significant (Table 4).

The parental origin of mutations was identified in 304 patients. Patients inheriting PKDl mutations from their fathers (paternal origin) showed shorter renal survival than 


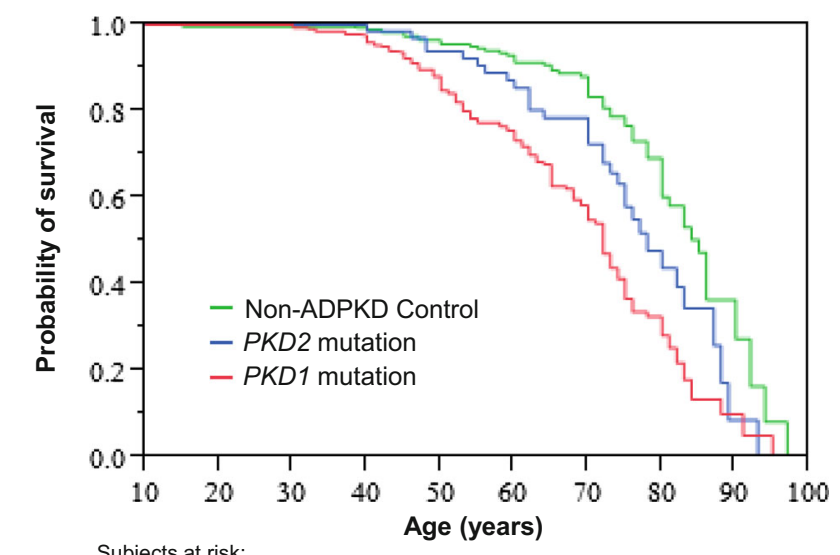

\begin{tabular}{r|cccccc} 
Control $(\mathrm{n}=301)$ & 297 & 275 & 208 & 169 & 113 & 46 \\
PKD2 mutation $(\mathrm{n}=72)$ & 72 & 71 & 60 & 51 & 39 & 12 \\
PKD1 mutation $(\mathrm{n}=338)$ & 338 & 305 & 223 & 162 & 84 & 23
\end{tabular}

Fig. 1 Kaplan-Meier analysis, showing survival curves of nonADPKD family members and of patients with PKD2 and PKD1 mutations. Mean \pm SE survival is significantly different among nonADPKD family members (81.7 \pm 1.2 years), patients with PKD2 mutations $(76.3 \pm 1.9$ years), and patients with PKD1 mutations $(69.7 \pm 1.1$ years) (log rank test, $P<0.0001) . P K D$ genic mutations severely affect patient survival, as well as renal survival

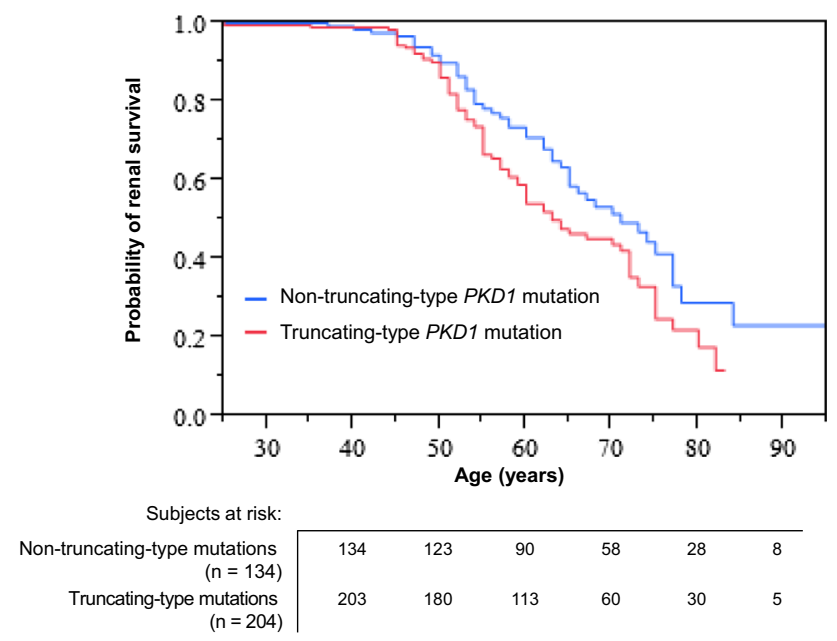

Fig. 2 Renal survival plots of carriers of $P K D 1$ non-truncating- and truncating-type mutations. $P K D 1$ mutation types affect renal survival (log rank test, $P=0.0348$ ). The mean age difference is 4.3 years. The different renal survival is explained by the complete or incomplete inactivation of $P K D 1$

those inheriting $P K D 1$ mutations from their mothers (maternal origin) (log rank test, $P=0.0039$; Table 4).

\section{Discussion}

The identification of pathogenic mutations by using a combination of NGS, LR-PCR, MLPA, and an analysis software package provided an overall $P K D$ mutation detection rate of $93.1 \%(94 / 101)$ [23]. This detection rate was similar (84.5-93.8\%) to that reported in recent largescale $P K D$ studies $[5,7,8,10,12]$.

$P K D 1$ and $P K D 2$ pathogenic mutations accounted for 81.2 and $11.9 \%$ of pedigrees, respectively (Table 1 ). These percentages of patients with $P K D 1$ and $P K D 2$ mutations are not significantly different from those reported in previous studies $[5,7,8,10,12]$. The ratio of patients with PKD1 to PKD2 mutations found by recent gene analyses seems approximately uniform, despite different ethnic groups, when the study avoids the preferential inclusion or exclusion of patients receiving RRT. In patients with $P K D 1$ mutations, $59.8 \%$ of pedigrees exhibited truncating-type mutations (Table 1). This percentage was slightly lower than that reported in previous studies (65.1-69.9\%), but the difference was not statistically significant $[5,7,10]$.

Renal survival was reported to be longer by approximately 20 years in patients with $P K D 2$ mutations than in patients with $P K D 1$ mutations $[8,11,26]$. In this study, the mean and median ages of patients with $P K D 1$ mutations, at the onset of ESRD, were 66.9 and 67 years (95\% CI 63-72), respectively. The median ages at the onset of ESRD in the Catalan [11], European [26], and Genkyst [8] studies were 53.4, 54.3, and 58.1 years, respectively. The mean renal survival in patients with $P K D 2$ mutations was 75.2 years in the present study (Table 3 ), which was within the reported range (69.1-79.7 years) [8, 11, 26].

The age at the onset of ESRD increased in later studies. An improved renal prognosis after approximately 8 years was observed in patients with ADPKD [27]. According to the annual report of the Japanese Society for Dialysis Therapy, the mean age of patients with ADPKD at the onset of dialysis therapy was 54.8 years in 1987, and increased to 63.1 years in 2014 [28]. The delayed onset of dialysis therapy was generally recognized in other chronic kidney diseases. The shorter interval, at the onset of ESRD, between patients with $P K D 1$ and $P K D 2$ mutations, in the present study, might be explained by the preferential improvement of the renal prognosis in patients with worse kidney function, such as patients with $P K D 1$. In addition, the 5-10\% lower fraction of truncating-type mutations in these patients (Table 1) might explain, in part, their longer renal survival. The factors mentioned above (renal survival was improved in recent reports and relatively small percentage of patients with truncating-type mutation) might partly explain the longer renal survival in patients with PKD1 mutation of this study than those reported previously $[8,11,26]$. Other factors might be possible and large number study is needed.

Most studies involving a relatively large population have reported improved renal survival in women compared to that in men $[4,8,10,29]$; however, some studies did not report this sex-related difference in patients with $P K D 1$ mutations $[13,26]$. The tendency of favorable renal 


\section{A PKD1 truncating-type mutations}

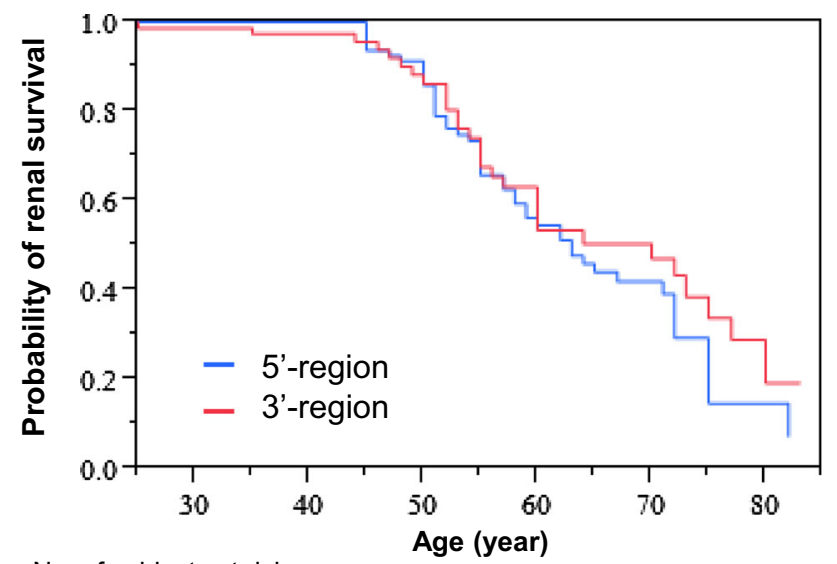

No. of subjects at risk

\begin{tabular}{l|cccccc}
\cline { 2 - 6 } 5'-region & 125 & 115 & 68 & 34 & 15 & 3 \\
3'-region & 78 & 65 & 45 & 26 & 15 & 3
\end{tabular}

Fig. 3 Position $\left(5^{\prime}\right.$ - versus $3^{\prime}$-region, divided at nucleotide \#7978) of a truncating- and $\mathbf{b}$ non-truncating-type mutations, showing different effects on renal survival. b The position of non-truncating-type mutations has a significant influence (log rank test, $P=0.0014$ ), whereas a that of truncating-type mutations does not have a

\section{B PKD1 non-truncating-type mutations}

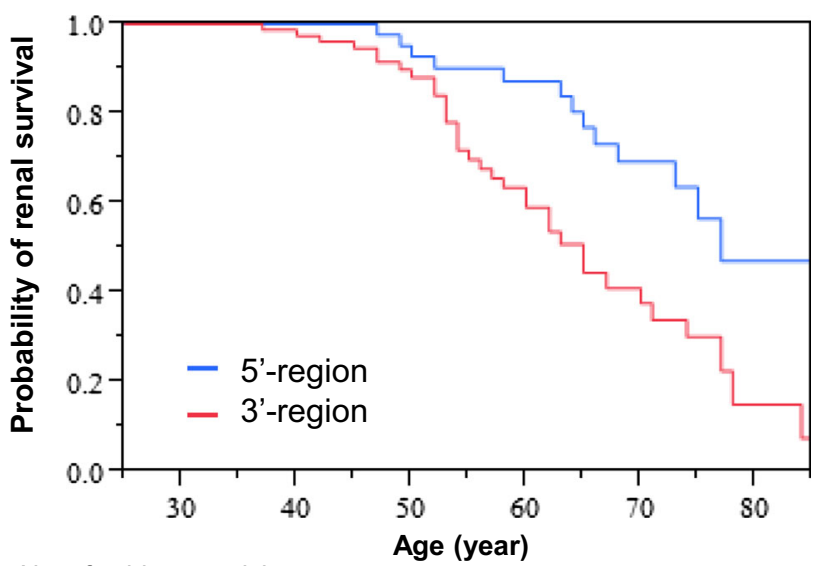

No. of subjects at risk

\begin{tabular}{l|llllll}
\cline { 2 - 7 } 5'-region & 49 & 47 & 39 & 29 & 16 & 5 \\
3'-region & 85 & 76 & 51 & 29 & 12 & 3
\end{tabular}

significant influence ( $\log$ rank test, $P=0.4375$ ). This difference might be due to the uniform inactivation of the CTT domain, irrespective of the position of the mutation in a, compared to the variability of CTT inactivation in $\mathbf{b}$, based on the location of mutation sites. See Fig. 4

Table 4 Effects of gender and parental origin on renal survival

\begin{tabular}{|c|c|c|c|c|c|c|c|}
\hline \multirow[t]{2}{*}{ Variables } & \multirow[t]{2}{*}{ Subjects $(n)$} & \multicolumn{3}{|c|}{ Survival (years) by Kaplan-Meier analysis } & \multicolumn{3}{|c|}{ Cox's proportional hazards analysis } \\
\hline & & Mean & SE & $P$ value & Univariate HR & $95 \% \mathrm{CI}$ & $P$ value \\
\hline \multicolumn{8}{|l|}{ Gender } \\
\hline \multicolumn{8}{|l|}{ All $P K D$} \\
\hline Female & 216 & 70.25 & 1.10 & Log rank test, $P=0.1729$ & 1 (referent) & & \\
\hline Male & 194 & 68.68 & 1.32 & Wilcoxon test, $P=0.2022$ & 1.26 & $0.90-1.78$ & 0.1800 \\
\hline \multicolumn{8}{|l|}{$P K D 1$} \\
\hline Female & 179 & 67.67 & 1.28 & Log rank test, $P=0.1444$ & 1 (referent) & & \\
\hline Male & 159 & 65.32 & 1.41 & Wilcoxon test, $P=0.1866$ & 1.29 & $0.91-1.84$ & 0.1524 \\
\hline \multicolumn{8}{|l|}{$P K D 2$} \\
\hline Female & 37 & 74.96 & 1.05 & Log rank test, $P=0.4677$ & 1 (referent) & & \\
\hline Male & 35 & 67.64 & 0.50 & Wilcoxon test, $P=0.5837$ & 0.56 & $0.08-2.44$ & 0.4592 \\
\hline \multicolumn{8}{|c|}{ Parental origin } \\
\hline \multicolumn{8}{|l|}{ All $P K D$} \\
\hline Maternal & 180 & 69.80 & 1.25 & Log rank test, $P=0.1743$ & 1 (referent) & & \\
\hline Paternal & 124 & 65.62 & 1.36 & Wilcoxon test, $P=0.0565$ & 1.31 & $0.88-1.96$ & 0.1831 \\
\hline \multicolumn{8}{|l|}{$P K D 1$} \\
\hline Maternal & 149 & 67.93 & 1.43 & Log rank test, $P=0.0039$ & 1 (referent) & & \\
\hline Paternal & 99 & 61.53 & 1.46 & Wilcoxon test, $P=0.0104$ & 1.84 & $1.20-2.83$ & 0.0054 \\
\hline \multicolumn{8}{|l|}{$P K D 2$} \\
\hline Maternal & 31 & 68.77 & 0.95 & Log rank test, $P=0.1444$ & 1 (referent) & & \\
\hline Paternal & 25 & 76.11 & 1.23 & Wilcoxon test, $P=0.1222$ & 0.33 & $0.05-1.42$ & 0.1417 \\
\hline
\end{tabular}

Parental origin could not be determined in 106 patients

$E S R D$ end-stage renal disease 


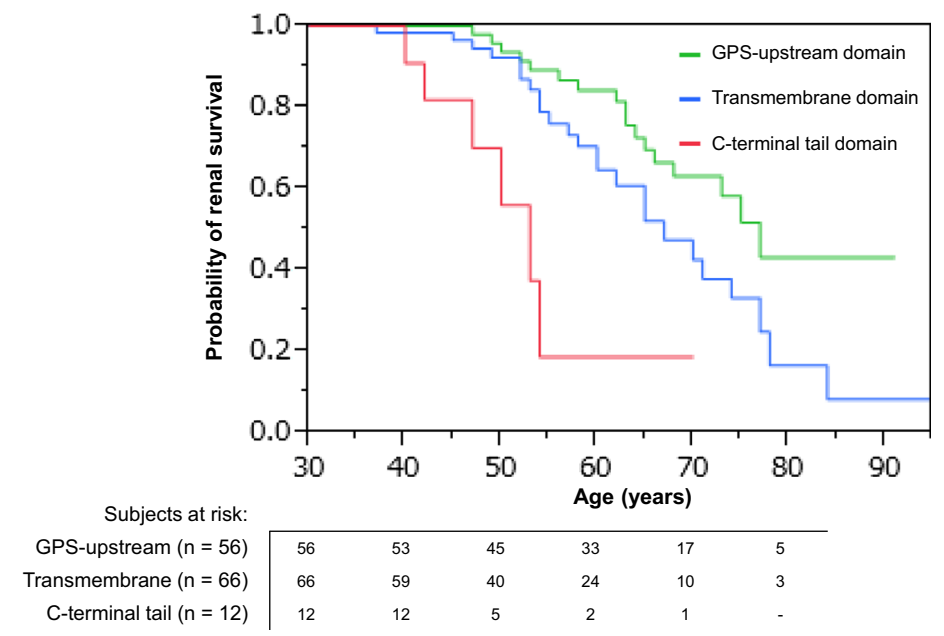

Fig. 4 Kaplan-Meier analysis, showing renal survival curves of patients with PKD1 non-truncating-type mutations in the GPSupstream domain, transmembrane domain, and CTT domain. Mean \pm SE renal survival is significantly different among patients with non-truncating-type mutations in the GPS-upstream domain
$(70.2 \pm 1.5$ years $)$, in the transmembrane domain $(67.0 \pm 2.2$ years $)$, and in the CTT domain $(50.1 \pm 1.7$ years) (log rank test, $P<0.0001)$. This figure indicates a possible crucial role of the CTT domain in PC1 function

renal survival plots comparing PKD1 mutations in the $5^{\prime}$ and $3^{\prime}$-regions did not show any significant difference $(P=0.69)$ in another study [8], and in the present study (log rank test, $P=0.2263$; Table 3 ). This difference might be partly explained by improvements in both molecular analyses and the scoring of missense mutations in the last 10 years, which resulted in identification of a significant number of mild or "hypomorphic" alleles [8]. Our observations support this report.

The influence of the position of the mutation on renal survival was strikingly different between patients with truncating- and non-truncating-type PKDl mutations (Table 3; Fig. 3). This influence was significant based on Cox's hazard analysis in patients with non-truncating-type $P K D 1$ mutations $(P=0.0013$; Table 3$)$, but was not significant in patients with truncating-type $P K D 1$ mutations $(P=0.4452$; Table 3$)$. The differences in the renal phenotypes between carriers of $3^{\prime}$-end non-truncating-type mutations and carriers of $5^{\prime}$-end non-truncating-type mutations was confirmed by the differences in renal survival among carriers harboring mutations in three distinct domains. The renal prognosis for carriers of non-truncating-type PKD1 mutations became more severe in the following order: GPS-upstream, transmembrane, and CTT domain mutations (Table 3; Fig. 4; Supplemental Fig. 3). These findings might be in accordance with the essential role of $\mathrm{PC} 1-\mathrm{PC} 2$ complex formation through the interaction of their CTT domains for mechanosensory PC function in the primary cilium [16-18]. Topological relationship among three domains, equal group mutation position around median nucleotide \#7978 and cDNA midpoint (nucleotide \#6456) is illustrated in Supplemental Fig. 3. 
Dissimilar $P K D 1$ allelic influences between truncating and non-truncating mutations were explained by the complete inactivation of $\mathrm{PC} 1$ in truncating-type mutations $[8,10]$. The complete inactivation of PC1 may not be affected by positional differences ( $3^{\prime}$ vs $5^{\prime}$ ), with respect to the expressed phenotype. In non-truncating-type mutations, on the other hand, the variability in renal survival due to positional differences may be recognized, due to the incomplete inactivation of PC1 (Table 3; Fig. 3).

However, the results of the present study provide additional insights into the dissimilar allelic influence on the renal phenotype. Irrespective of the position of the mutation (in the $3^{\prime}$ - or $5^{\prime}$-region), the inactivation of the CTT domain is expected in truncating-type mutations. Hence, differences in renal survival were not observed between patients with mutations in the $3^{\prime}$ - or the $5^{\prime}$-region. In addition, the inactivation of the CTT domain is heterogeneous, based on the positions of the mutations, in nontruncating-type mutations. Hence, a milder phenotype is expressed in non-truncating-type mutation carriers than in truncating-type mutation carriers, due to the reduced inactivation of the CTT domain in non-truncating-type mutations.

The influence of mutated loci on renal survival has been rarely reported in patients with ADPKD. A possible correlation between the mutated loci and the disease phenotype has been reviewed in patients with cystic fibrosis [30]. However, additional genotype and phenotype correlation studies are needed to explore the integrated relationships between mutation types and positions in patients with ADPKD [31-33].

A limitation of this study is the inclusion of a relatively small number of patients, compared with that in large-scale $P K D$ studies $[8,10]$. However, the present study confirmed the significant allelic influence of PKDl mutation types on renal survival, which is consistent with that reported in previous large-scale studies $[8,10]$. In addition, our results suggested a potentially crucial role for the CTT domain in renal prognosis, which requires a more comprehensive elucidation in future larger scale studies.

\section{Compliance with ethical standards}

Funding Otsuka Pharmaceutical Co., Ltd., provided funding for performing this study.

Conflict of interest Employment: $\mathrm{MK}$ is an employee of Otsuka Pharmaceutical. Grants received: EH and SH received funding from Otsuka Pharmaceutical. Other authors declare that they do not have any conflicts of interest directly relevant to the contents of this manuscript.

Human and animal rights Informed consent was obtained from all individual participants included in the study. The study protocol adhered to the Declaration of Helsinki, and was approved by the institutional review boards of Kyorin University and Juntendo University (nos. 579 and 14-043, respectively).

Registration of clinical trials This study is registered in ClinicalTrials.gov under accession no. NCT02322385.

Open Access This article is distributed under the terms of the Creative Commons Attribution 4.0 International License (http://crea tivecommons.org/licenses/by/4.0/), which permits unrestricted use, distribution, and reproduction in any medium, provided you give appropriate credit to the original author(s) and the source, provide a link to the Creative Commons license, and indicate if changes were made.

\section{References}

1. Dalgaard OZ. Bilateral polycystic disease of the kidneys; a follow-up of two hundred and eighty-four patients and their families. Acta Med Scand. 1957;28:S1-255.

2. Churchill DN, Bear JC, Morgan J, Payne RH, McManamon PJ, Gault MH. Prognosis of adult onset polycystic kidney disease reevaluated. Kidney Int. 1984;26:190-3.

3. Gabow PA, Johnson AM, Kaehny WD, et al. Factors affecting the prognosis of renal disease in autosomal dominant polycystic kidney disease. Kidney Int. 1992;41:1311-9.

4. Higashihara E, Nutahara K, Kojima M, et al. Prevalence and renal prognosis of diagnosed autosomal dominant polycystic kidney disease in Japan. Nephron. 1998;80:421-7.

5. Rossetti S, Consugar MB, Chapman AB, et al. Comprehensive molecular diagnostics in autosomal dominant polycystic kidney disease. J Am Soc Nephrol. 2007;18:2143-60.

6. Barua M, Cil O, Paterson AD, et al. Family history of renal disease severity predicts the mutated gene in ADPKD. J Am Soc Nephrol. 2009;20:1833-8.

7. Audrézet MP, Cornec-Le Gall E, Chen JM, et al. Autosomal dominant polycystic kidney disease: comprehensive mutation analysis of PKD1 and $P K D 2$ in 700 unrelated patients. Hum Mutat. 2012;33:1239-50.

8. Cornec-Le Gall E, Audrézet MP, Chen JM, et al. Type of PKD1 mutation influences renal outcome in ADPKD. J Am Soc Nephrol. 2013;24:1006-13.

9. Kurashige M, Hanaoka K, Imamura M, et al. A comprehensive search for mutations in the PKD1 and PKD2 in Japanese subjects with autosomal dominant polycystic kidney disease. Clin Genet. 2015;87:266-72.

10. Heyer CM, Sundsbak JL, Abebe KZ, et al. Predicted mutation strength of nontruncating PKDl mutations aids genotype-phenotype correlations in autosomal dominant polycystic kidney disease. J Am Soc Nephrol. 2016;27:2872-84.

11. Torra R, Badenas C, Darnell A, et al. Linkage, clinical features, and prognosis of autosomal dominant polycystic kidney disease types 1 and 2. J Am Soc Nephrol. 1996;7:2142-51.

12. Hwang YH, Conklin J, Chan W, et al. Refining genotype-phenotype correlation in autosomal dominant polycystic kidney disease. J Am Soc Nephrol. 2016;27:1861-8.

13. Rossetti S, Burton S, Strmecki L, et al. The position of the polycystic kidney disease $1(P K D 1)$ gene mutation correlates with the severity of renal disease. J Am Soc Nephrol. 2002;13:1230-7.

14. The European Polycystic Kidney Disease Consortium. The polycystic kidney disease 1 gene encodes a $4 \mathrm{~kb}$ transcript and lies within a duplicated region on chromosome 16. Cell. 1994;77:881-94. 
15. Hughes J, Ward CJ, Peral B, et al. The polycystic kidney disease 1 (PKD1) gene encodes a novel protein with multiple cell recognition domains. Nat Genet. 1995;10:151-60.

16. Chapin HC, Caplan MJ. The cell biology of polycystic kidney disease. J Cell Biol. 2010;191:701-10.

17. Gainullin VG, Hopp K, Ward CJ, Hommerding CJ, Harris PC. Polycystin-1 maturation requires polycystin- 2 in a dose-dependent manner. J Clin Investig. 2015;25:607-20.

18. Kim $\mathrm{H}, \mathrm{Xu} \mathrm{H}$, Yao Q, et al. Ciliary membrane proteins traffic through the Golgi via a Rabep1/GGA1/Arl3-dependent mechanism. Nat Commun. 2014;5:5482.

19. Yu S, Hackmann K, Gao J, et al. Essential role of cleavage of polycystin-1 at $\mathrm{G}$ protein-coupled receptor proteolytic site for kidney tubular structure. Proc Natl Acad Sci USA. 2007; 104:18688-93.

20. Chauvet V, Tian X, Husson H, et al. Mechanical stimuli induce cleavage and nuclear translocation of the polycystin-1 $\mathrm{C}$ terminus. J Clin Investig. 2004;114:1433-43.

21. Low SH, Vasanth S, Larson CH, et al. Polycystin-1, STAT6, and P100 function in a pathway that transduces ciliary mechanosensation and is activated in polycystic kidney disease. Dev Cell. 2006; 10:57-69.

22. Pei Y, Obaji J, Dupuis A, et al. Unified criteria for ultrasonographic diagnosis of ADPKD. J Am Soc Nephrol. 2009;20:205-12.

23. Kinoshita M, Higashihara E, Kawano $\mathrm{H}$, et al. Identification of pathogenic mutations in $P K D 1$ and $P K D 2$ in patients with autosomal dominant polycystic kidney disease by next-generation sequencing and use of a comprehensive new classification system. PLoS One. 2016;11:e0166288.
24. UniProt Consortium. UniProt: a hub for protein information. Nucleic Acids Res. 2015;43:D204-12.

25. Wilson PD. Polycystin: new aspects of structure, function, and regulation. J Am Soc Nephrol. 2001;12:834-45.

26. Hateboer N, v Dijk MA, Bogdanova N, et al. Comparison of phenotypes of polycystic kidney disease types 1 and 2 . European PKD1-PKD2 Study Group. Lancet. 1999;353:103-7.

27. Schrier RW, McFann KK, Johnson AM. Epidemiological study of kidney survival in autosomal dominant polycystic kidney disease. Kidney Int. 2003;63:678-85.

28. Nakamoto H, Masakane I. An overview of regular dialysis treatment in Japan as of Dec. 31, 2015. Tokyo: The Japanese Society for Dialysis Therapy; 2016. p. 1-70 (in Japanese).

29. Harris PC, Bae KT, Rossetti S, et al. Cyst number but not the rate of cystic growth is associated with the mutated gene in autosomal dominant polycystic kidney disease. J Am Soc Nephrol. 2006;17:3013-9.

30. Rowntree RK, Harris A. The phenotypic consequences of CFTR mutations. Ann Hum Genet. 2003;67:471-85.

31. Harris PC, Rossetti S. Determinants of renal disease variability in ADPKD. Adv Chronic Kidney Dis. 2010;17:131-9.

32. Torres VE, Harris PC, Pirson Y. Autosomal dominant polycystic kidney disease. Lancet. 2007;369:1287-301.

33. Rossetti S, Chauveau D, Kubly V, et al. Association of mutation position in polycystic kidney disease 1 (PKD1) gene and development of a vascular phenotype. Lancet. 2003;361:2196-201. 